\title{
Adaptive Control Program for Rough Turning Machining Processes
}

\author{
Samy Oraby ${ }^{1}$ and Ayman Alaskari ${ }^{2}$
}

\begin{abstract}
Although much research have been carried out to study and evaluate theory of metal cutting and machining, unsatisfactory repetitive outcome was obtained with a wide domain of variability. Although numerical control (NC) technology of machine tools has contributed to the machining topic in terms of more flexibility, better surface quality and dimensional accuracy, and higher productivity, it still incapable to adapt to the dynamic conditions that result from continuous variations during cutting. Current CNC machines follow preprogrammed fixed feeds and speeds during each cutting segment.

In contrast to NC procedures, adaptive control (AC) technique measures the process output (responses) in real time, and automatically adjusts and continuously tunes cutting feed and/or speed to the optimal levels during each operation so as to achieve some objectives under the imposed system constraints.

In the current work, an adaptive control simulation strategy is proposed in which the core of the optimization routine is based on some mathematical empirical models that define the interrelationship between the system responses (output) and the operating conditions (speed and feed). These models independently define the targeted primary objective; the metal removal rate (MRR), the secondary objectives; wear or its rate, and the system constraints; cutting forces and machining power. Optimization strategy involves the search for the best operational speed and/or feed combination that maximizes the MRR while attaining the lowest possible edge wear and/or its rate (tool life) under system constraints of tolerable force level and available spindle power. While all previously developed AC approaches addressed only the cutting feed and its relevant force level in milling, the proposed mathematical-model based adaptive control system deals with the turning operation where the cutting speed, as the main controlling parameter of the edge wear, along with the feed are considered.
\end{abstract}

Keywords-Adaptive Control, Cutting forces, Machining processes, Metal Removal Rate, Tool Wear.

\section{INTRODUCTION}

A LTHOUGH metal cutting and machining topic is as old as the beginning of the last century [1], it is still least understood. This is due the complex and unlimited interrelated frictional, thermal, and tribological parameters that are involved in the process [2]. Throughout the so far huge research studied the metal cutting and machining in addition to the dedicated machinability database sources, unsatisfactory repetitive outcome was obtained with a wide domain of variability [3] and [4].

Samy Oraby ${ }^{1}$ is with Dept. Manufacturing Engineering Technology, College of Technological Studies, PAAET, Kuwait.

Ayman Alaskari ${ }^{2}$ is with Dept. Manufacturing Engineering Technology, College of Technological Studies, PAAET, Kuwait.
Numerical control (NC) of machine tool has contributed to the machining topic in terms of more flexibility, better surface quality and dimensional accuracy, and higher productivity [5]. In $\mathrm{NC}$, the required part configuration is obtained through a part program containing specially dedicated syntax information to simply guide the machine servomotors to where and how to go to a specified target point. Such a part program is usually prepared in advance by qualified team with appropriate arithmetic and mathematical knowledge along with sufficient experience and skills regarding machining aspects along with machinability principles. Current CNC machines follow preprogrammed fixed feeds and speeds during each cutting segment. Therefore, they do not have the flexibility to adapt to the dynamic conditions that result from continuous variations during cutting. In most practical situations, cutting process varies unpredictably during machining due to the variation in cut depth and/or width; in the tool sharpness (tool wear and deformation); or, in composition homogeneity of the material being cut. Therefore, for safety considerations to avoid damages during machining, conservative permanent cutting parameters are usually preferred, resulting in inefficient machining performance. Alternatively, in an attempt to shorten cycle times, aggressive cutting parameters are sometimes selected, often resulting in catastrophic damage to tools, parts, and machines. With expected variations in the cutter performance due to wear and deformation, and in the consumed power and force load, human interruption is essentially required in terms of operation stops and program modifications. This is against the ultimate objectives of fully unmanned operation with minimum cost.

With the introduction of newly developed ultra-hard work materials with various metallurgical and mechanical properties that are machined by super-hard and sophisticated tool with complex configuration, the very rigid and powerful $\mathrm{NC}$ machines offer a unique way to machining as a manufacturing process. However, the search for robust and smart manipulation techniques is required to justify the high investment in the NC hardware, software and maintenance. The approach of in-process monitoring and diagnosis of the process and, accordingly taking the appropriate action may play such a crucial role.

Adaptive control (AC) of machine tools introduces a promising technique to optimize, monitor, and control machining process to achieve the fully automated system attaining maximum productivity. In contrast to NC part program operation, adaptive control techniques [6]-[11] are based on monitoring the process variables (responses) in real time, and automatically adjust and continuously tune cutting 
feed and/or speed to their optimal levels during each operation so as to achieve some objectives under the imposed system constraints. Measurable responses include cutting forces [6], [7], [9] and [11], and vibration [6]. Based on this approach, AC system improves metal removal rate MRR through reducing cycle time [7], maintains surface roughness for the machined surface [6] and [9] or prevents damage to tools, parts, and machine, and minimizing production disruptions [10] and [11].

However, many control systems and instrumentation problems are still the main obstacles delaying the establishment of a universally feasible commercial system. Of the very few emerged practical systems is the Adaptive Control Monitoring ACM ready-to-install technology offered by OMATIVE Systems (http://www.omative.com) [11]. OMATIVE ACM is claimed to automatically ensure that critical cutting measurable responses parameters do not exceed their acceptable levels. The system has the ability to warn of any impending damage and if necessary, it stops the machine in cases of overload conditions or tool breakage, Fig. 1.b. As shown by Fig. 1.b, instead of using a fixed programmed feed regardless variation in work configuration, feed rate is varied according to the variation in the measured spindle load due to air gabs, changing depth or width, etc. that, feed rate varies so as to suit the instantaneous situation.

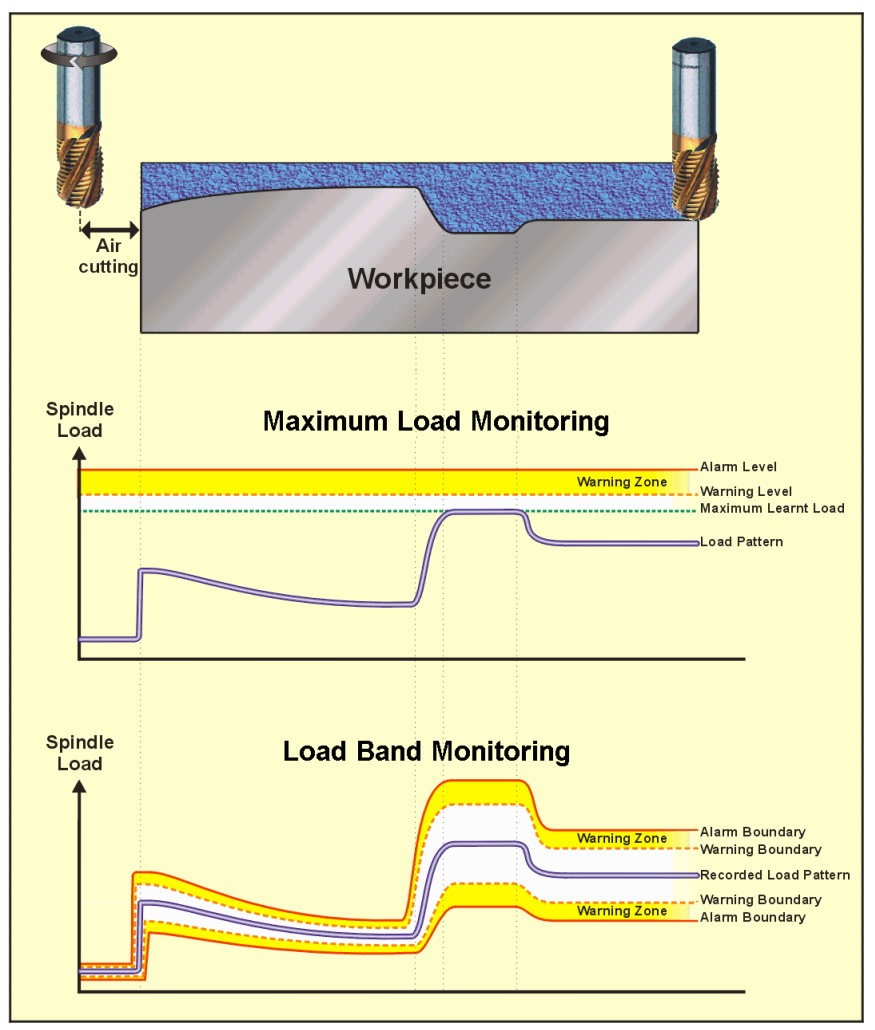

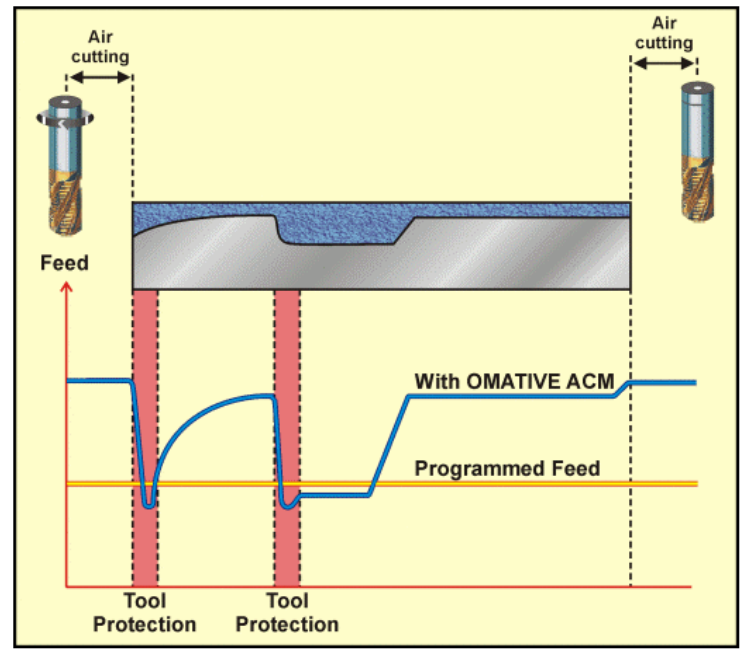

a) Load Monitoring Feed Optimization

Fig. 1 OMATIVE ACM system (courtesy OMATIVE Systems [11])

In the current work, an adaptive control simulation strategy is proposed in which the core of the optimization routine is based on some previously developed mathematical empirical group of models covering a definite domain of tool-workmachine combination. Operations objectives include maximizing the metal removal rate MRR, as a primary objective, while minimizing tool wear, or its rate as a secondary objectives. In other word the question to be addressed here is: Among the available cutting parameters what is the speed-feed combination to achieve the maximum removed metal while attaining the lowest possible edge wear and/or its rate. Prolonging tool life could be also considered as an objective in the case of employing costly cutting inserts. System constraints are the consumed spindle power, machine tool capacity, and the maximum allowable cutting forces.

While all previously developed AC approaches addressed the manipulation of only cutting feed in the end milling operations, the proposed mathematical-model based adaptive control system deals the rough turning process where the cutting speed usually has the most predominant influence on the edge wear and its rate [12].

\section{DEVELOPMENT OF MATHEMATICAL EMPIRICAL MODELS IN MACHINING}

Experimental data [13] were used to develop mathematical models using nonlinear regression procedures. Multicoated carbide inserts [Sandvik GC345] were used to machine an alloy steel 709M40 (En 19), under dry cutting conditions, using a Colchester Mascot centre lathe. A practical domain of operating parameters for rough turning was covered to include cutting speed (V) $50-200 \mathrm{~m} / \mathrm{min}$, feed (f) $0.06-0.6 \mathrm{~mm} / \mathrm{rev}$; and depth of cut (d) $1.5-3 \mathrm{~mm}$. For modeling significance, experiments were specified according to Central Composite Design CCD of the experiments led to 24 tests. For each experiment, two-minute interval subtests were carried out before the wear on the cutting edge was assessed at nose; flank; and notch. During each subtest three force components 
were measured, feed Fx, power Fy and radial Fz using threecomponent strain gauge dedicated dynamometer [14].

To establish an empirical functional interrelationship between a dependent variable or, response (R), and some dependent variables ( $\xi$,s), the first stage is to propose a firstorder model to start with taking the general form:

$$
\mathrm{R}=\mathrm{b}_{0}+\sum_{\mathrm{j}=1}^{\mathrm{p}} \mathrm{b}_{\mathrm{j}} \xi_{\mathrm{j}}+\varepsilon_{\mathrm{n}}
$$

where $\boldsymbol{\varepsilon}_{\mathbf{n}}$ is the error absolute value using linear nontransformed model.

The second stage is to promote the developed model to detect the possible nonlinear nature of the independent variables using parameters estimates from the first-order model as initial values in the nonlinear iterative least squares routine in the form:

$$
\mathrm{R}=\mathrm{c}_{\mathrm{O}} \quad \prod_{\mathrm{j}=1}^{\mathrm{p}} \xi_{\mathrm{J}}^{\beta \mathrm{j}} \varepsilon^{\wedge}
$$

in which $\xi_{\mathrm{J}}$ are the natural parameters, co and $\beta \mathrm{J}$ are the model parameters to be estimated using the experimental data, and $\varepsilon^{\wedge}$ is the multiplicative random error. In general natural form, this is characterized as:

$$
\mathrm{R}=\mathrm{c}_{\mathrm{o}} \mathrm{X}_{1}{ }^{\beta 1} \mathrm{X}_{2}{ }^{\beta 2} \mathrm{X}_{3}{ }^{\beta 3} \ldots \ldots \mathrm{X}_{\mathrm{J}}{ }^{\beta \mathrm{J}}
$$

Model coefficients co and $\beta \mathrm{J}$ are determined iteratively by the nonlinear regression procedures in association with the relevant experimental data.

In addition to the examination of residuals (zero mean, randomness, and probability normality), the adequacy and significant of the intended model is judged through the following criteria: $\mathrm{R}^{2}$, $\mathrm{t}$-statistic and, F-ratio.

According to the above strategy and procedures, the following models were established using the experimental data [13]:

Consumed_Power $(\mathrm{P})=0.140 \mathrm{~V}^{0.82} \mathrm{f}^{0.627} \mathrm{~d}^{0.844} \mathrm{t}^{-0.049} \mathrm{Aw}^{0.342}$

with $\mathrm{R}^{2}=0.97, \mathrm{SE}=0.062, \mathrm{~F}=4156$.

Thrust_Cutting_Force (Fxz) $=55354 \mathrm{~V}^{-0.484} \mathrm{f}^{0.236} \mathrm{~d}^{0.715} \mathrm{t}^{-0.173} \mathrm{Aw}$

with $\mathrm{R}^{2}=0.83, \mathrm{SE}=0.12, \mathrm{~F}=670$

Average_Tool_Wear $(\mathrm{Aw})=7.62 \mathrm{E}-03 \mathrm{~V}^{0.577} \mathrm{f}^{0.168} \mathrm{~d}^{0.248} \mathrm{t}^{0.245}$

with $\mathrm{R}^{2}=0.84, \mathrm{SE}=0.1, \mathrm{~F}=831$

Tool_Wear_Rate $(T W R)=7.62 \mathrm{E}-03 \mathrm{~V}^{0.577} \mathrm{f}^{0.168} \mathrm{~d}^{0.248} \mathrm{t}^{-0.755}$

In order to build a robust adaptive control AC strategy, the functional interrelationships between cutting responses (outputs such as force, power, tool wear, and metal removal rate) and the operating cutting parameters (speed-feed combination) should be clearly realized, Fig. 2. While cutting speed $\mathrm{V}$ seems to more activate edge wear and its rate than the feed f, Figs. 2.a \& 2.c, a reverse trend is observed as far as the thrust force $\mathrm{F}_{\mathrm{xz}}$ and metal removal rate MRR are concerned,
Figs. 2.a \& 2.e. Cutting speed and feed seem to have an equal influence of the consumed spindle power.

Based on these knowledge and considerations, the proposed approach is next described.

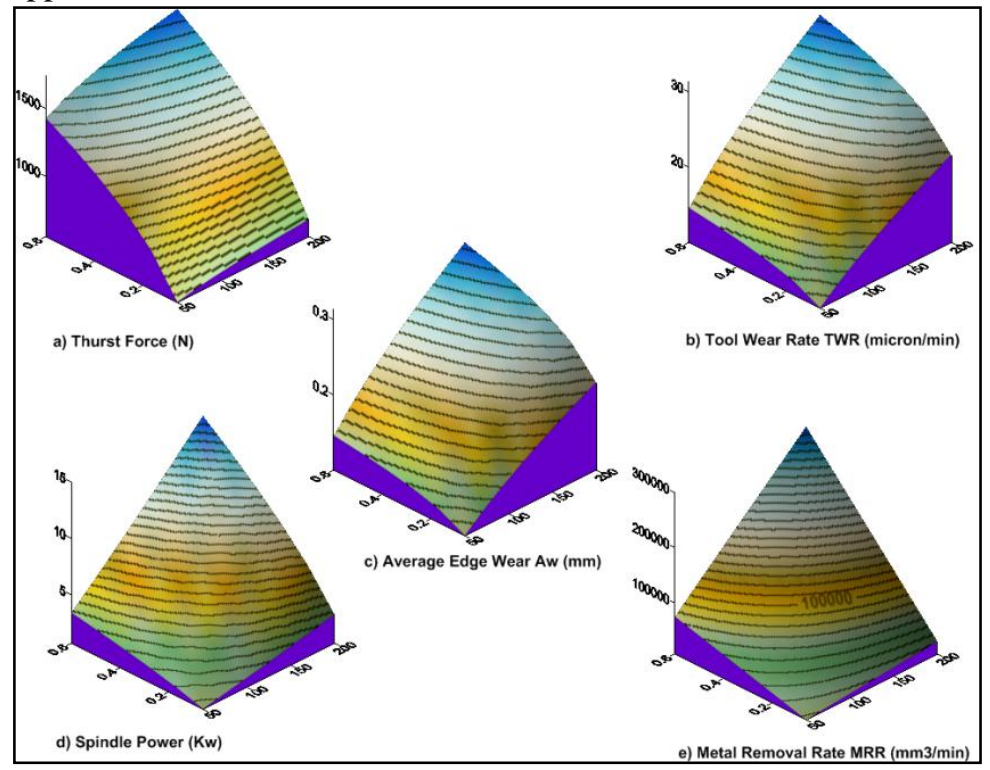

Fig. 2 Response surfaces of the different machining outputs

\section{Math Multi-Responses Multi-Constraints AC STRATEGY}

\section{A. Qualitative Descriptive Approach}

The proposed AC strategy based on mathematical models can be inserted in the host computer of CNC machine tools. With the appropriate operating and controlling syntax, the procedures may be schematically explained as shown in Fig. 3.

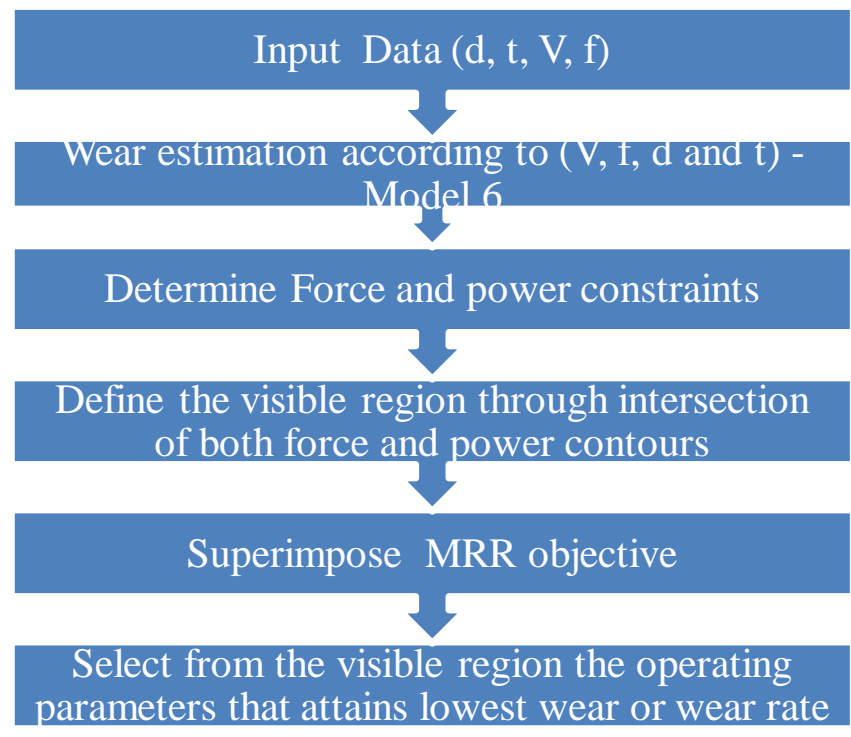

Fig. 3 Proposed AC mathematical models based adaptive control strategy

In the current proposed AC system, depth of cut is kept constant $(\mathrm{d}=2.5 \mathrm{~mm})$ as it is a work configuration dimension in rough longitudinal turning. However, whenever the need 
arises to change depth of cut, input data buffer should be updated to suit the latest value.

At any elapsed time, for instance $t=10 \mathrm{~min}$, the wear level is determined according to eq. 6 . Substitution of such value into each of power $\mathrm{P}$ and thrust force eqns. 4 and 5 yields the following equations:

$$
\text { Power }(P)=0.264 V^{1.0173} f^{0.6845} d^{0.9288} t^{0.0348}
$$

$$
\text { Force }(\text { Fxz })=259.0 \mathrm{~V}^{0.1507} \mathrm{f}^{0.4208} \mathrm{~d}^{1.243} \mathrm{t}^{0.0965}
$$

Considering depth $\mathrm{d}=2.5 \mathrm{~mm}$ and time $\mathrm{t}=10 \mathrm{~min}$, these become:

$$
\begin{gathered}
\text { Consumed_Power }(\mathrm{P})=0.067 \mathrm{~V}^{1.0173} \mathrm{f}^{0.6845} \\
\text { Thrust_Force }(\text { Fxz })=799.62 \mathrm{~V}^{0.1507} \mathrm{f}^{0.4208}
\end{gathered}
$$

Response surfaces for such force and power constraints are superimposed at the constraint power limit $(\mathrm{P}=7 \mathrm{Kw})$ and thrust force limit $\left(F_{x z}=1300 N\right)$, Fig. 4.a. Surfaces are intersected yielding the feasible boundaries ABCDEFA. Since such technique is rather a qualitative measure, graph contours are used by superimposing as shown in Fig. 4.b. The feasible ABCDEFA region is defined accurately in the speed-feed domain where power contour graph is superimposed on the force one. As shown by the visible region, infinite sets of feedfeed combinations are eligible to satisfy feasibility and, therefore, the only of those which benefits production objective must be searched for.

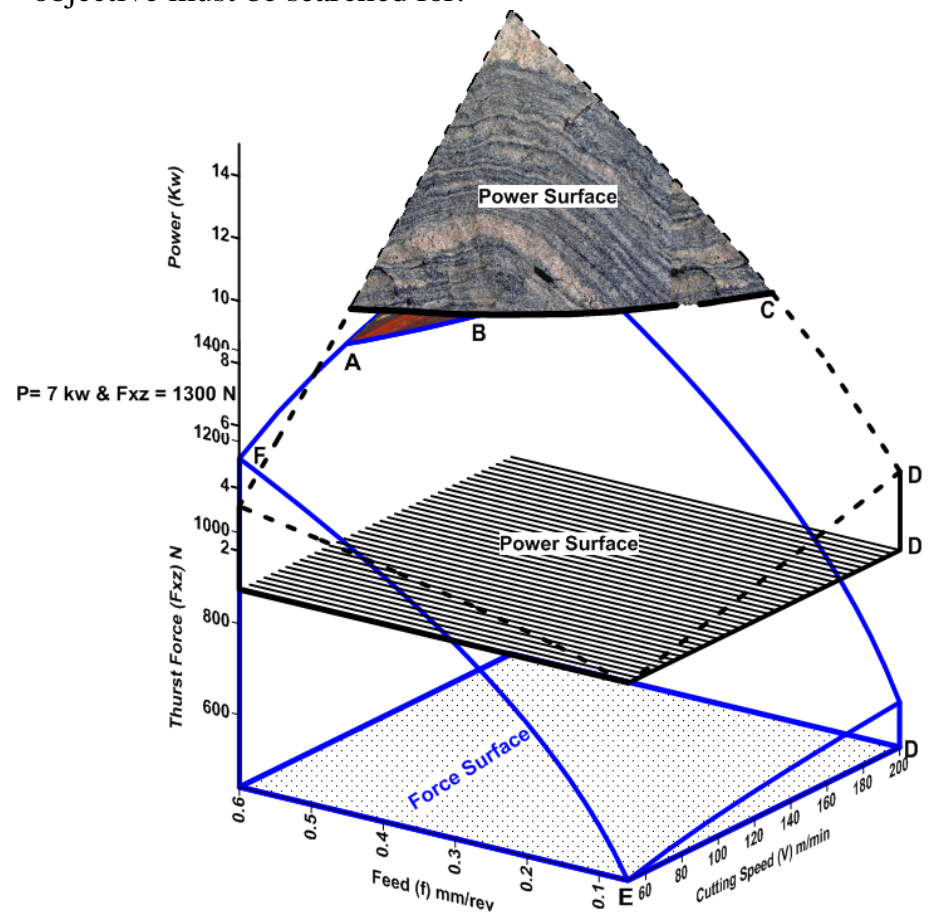

a) Force-Power surface intersection

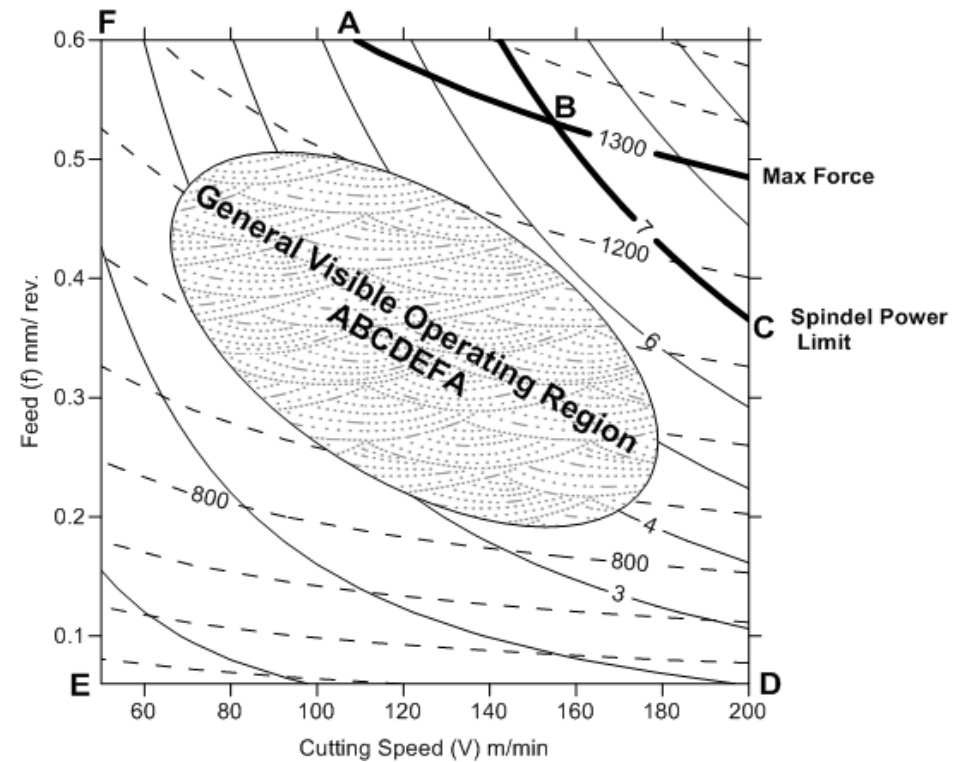

b) Contour graph of Force-Power intersection projection

Fig. 4 Visible operating domain according to force and power constraints

Next, the maximum metal removal rate MRR as a primary objective, calculated by eq. 12, is determined as shown in Fig. 5 as its contours are superimposed on the previously determined visible region.

$$
\begin{aligned}
\text { Metal_Removal_Rate }(\mathrm{MRR}) & =1000 \times \mathrm{V} \times \mathrm{f} \times \mathrm{d} \\
& =2500 \times \mathrm{V} \times \mathrm{f} \quad(\mathrm{d}=2.5 \mathrm{~mm})
\end{aligned}
$$

According to Fig. 5, the primary objective is attained as a max MRR of about $(205000 \mathrm{~mm} 3 / \mathrm{min}$ ) is obtained (point B on the boundary of ABCDEFA visible domain) considering the system under investigation. Such a unique point refers to a cutting parameters combination of $(154.7 \mathrm{~m} / \mathrm{min})$ speed and $(0.53 \mathrm{~mm} / \mathrm{rev})$ feed, Fig. 5 . Since point (B) is the boundary of the outlined visible domain, this implicates that it is impossible to obtain a higher MRR using the machining system under consideration.

However, the achieved primary objective ignores the secondary objective which is the state of the cutting edge. In many situations, wear and deformation on the cutting edge is functionally important not only from tool cost and its changing time considerations but also from safety point of views.

In order to compromise preserving cutting edge with some reduction in the MRR, the least tool wear rate TWR, eq. 13, within the visible region is selected as point $\mathrm{H}$, Fig. 5 .

$$
\text { Tool_Wear_Rate }(\mathrm{TWR})=1.681 \mathrm{~V}^{0.577} \mathrm{f}^{0.168}(\mu \mathrm{m} / \mathrm{min})
$$

This corresponds to parameter combination of $144 \mathrm{~m} / \mathrm{min}$ and $0.54 \mathrm{~mm} / \mathrm{revs}$ cutting speed and feed respectively, Fig. 6 . Such condition ensures TWR reduction from 27 to just 26 $\mu \mathrm{m} / \mathrm{min}$ for a decrease in the MRR from 205000 to 195000 $\mathrm{mm} 3 / \mathrm{min}$. In general, it can be define the region BGH as the visible region to determine the practical speed-feed range as: $144 \leq \mathrm{V} \leq 155 \mathrm{~m} / \mathrm{min} \& 0.53 \leq \mathrm{f} \leq 0.54 \mathrm{~mm} / \mathrm{rev}$. 
Depending on the dodge "Why to pay more while you could get perhaps cheaper?" or, on the base of "Why pay with no benefit?", there is no justification to consider any cutting conditions within BGH where speed is greater than $144 \mathrm{~m} / \mathrm{min}$ and less than about $0.5 \mathrm{~mm} / \mathrm{rev}$ inclusive.

When the minimum edge average wear Aw, eq. 14, is considered as a secondary objective, the same strategy is followed to yield the same operating cutting parameters, Fig. 7. Again, level of edge wear may be arbitrary reduced from 0.27 to $0.26 \mathrm{~mm}$ through a margin reduction in the MRR using a cutting combination of $147 \mathrm{~m} / \mathrm{min}$ cutting speed and 0.54 $\mathrm{mm} / \mathrm{rev}$ cutting feed rate. This implies that employing higher values of cutting speed or/and feed rate definitely leads to unjustified higher edge wear level in return of small increase in MRR. An additional positive side effect is that the reduction in the cutting parameters, especially speed, usually leads to a reduction in the cutting force and, consequently, in the consumed power.

However, the decision should be taken by the user according to entire group of factors affecting the process. This determines the principal priority to be set into the proposed strategy. If the tool cost is high, tool wear and its rate should be accounted for as a secondary objective without great deterioration in the process productivity or, the metal removal rate MMR. Beside, the system should be automatically tuned as any of the system constraints is changed positively or negatively.

Average_Tool_Wear $(\mathrm{Aw})=0.01681 \mathrm{~V}^{0.577} \mathrm{f} 0.168$

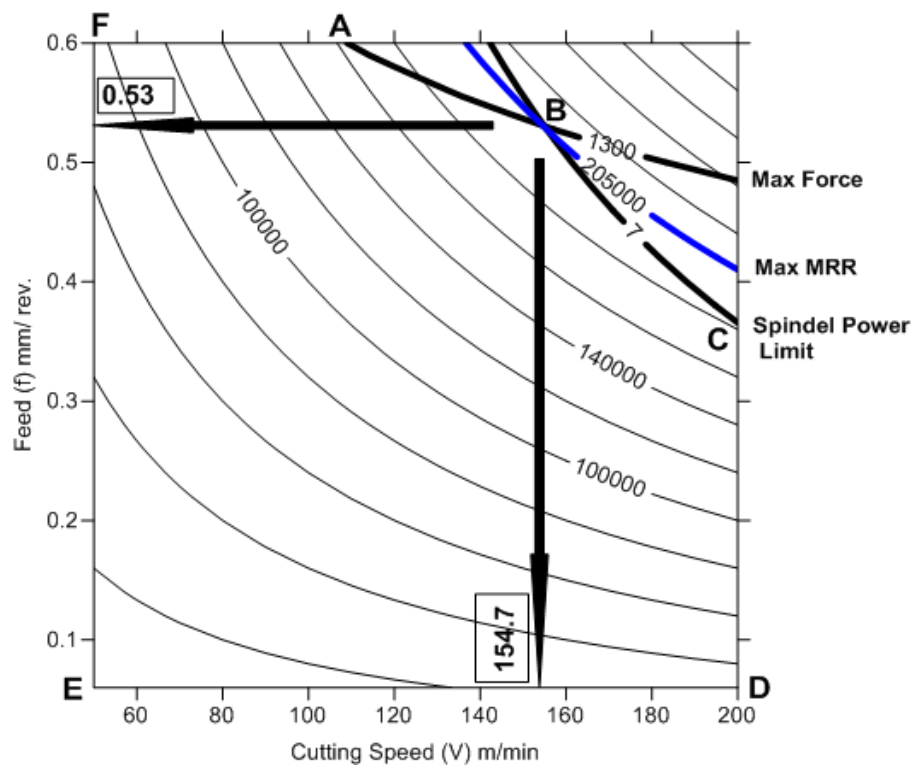

Fig. 5 Visible operating region and cutting parameters considering max MRR primary objective at force and power constraints

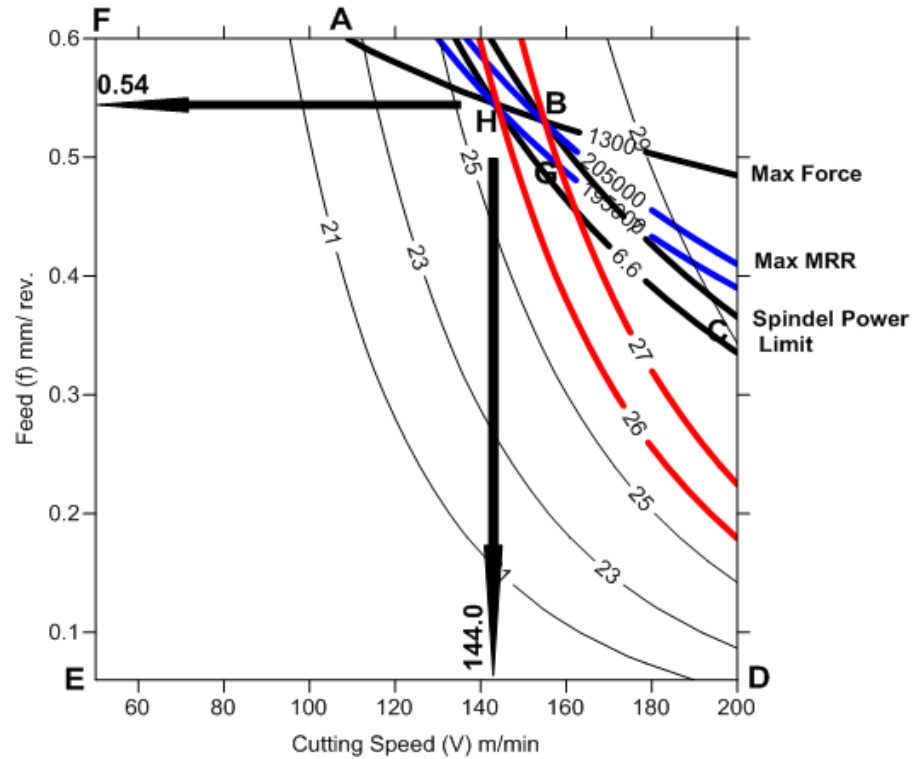

a) Entire Domain

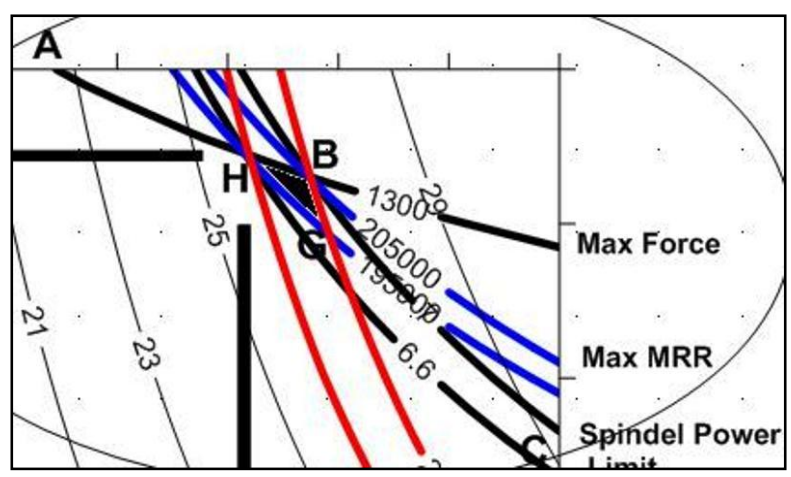

b) Zooming at the optimal region $\mathrm{BGH}$

Fig. 6 Best operating cutting conditions to achieve max MRR and minimum TWR under force and power constraints

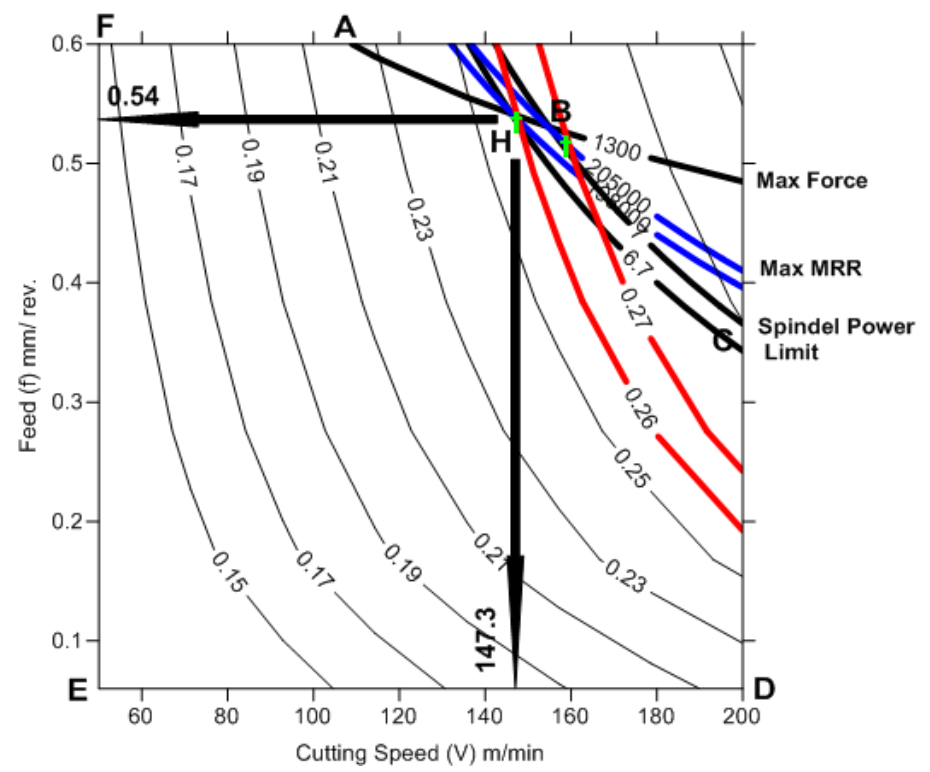

Fig. 7 Best operating cutting conditions to achieve max MRR and minimum edge wear Aw under force and power constraints 


\section{CONCLUSION}

In NC technology, machining begins and ends with fixed speed and feed operating parameters and, accordingly many practical and technical problems pertain. As the machining process continues, progressive edge wear develops leading to changes in all machining responses and outputs. Therefore, initial cutting conditions will be no longer valid. This either reduces operation productivity if conservative motion values are considered or jeopardized the safety of machining system and operators. Direct measuring of edge wear has not yet possible and therefore, process performance usually requires extra monitoring attention that leads to much time and money wasting without reaching the desired ultimate target of fully automated manufacturing processing.

An adaptive control mathematical models based strategy is proposed in the current study to monitor and o control the turning rough machining operation. The target is to achieve maximum metal removal rate through the selection of the instantaneous best speed and feed operating parameters and, not to violate system safety and its power capability.

At specified cut interval, the edge wear is assessed using the appropriate relevant empirical models and accordingly, the new cutting parameters are generated that ensure the best MRR without violation of the imposed forces and power constraints.

Analysis indicated a gain of at least 5\% in MRR over its value in comparison if fixed speed-feed is used instead. Additionally, the proposed strategy allows for the safer environment than if random operating parameters were rather employed.

\section{ACKNOWLEDGMENT}

The authors wish to thank the Public Authority for Applied Education and Training (PAAET) for funding this research under the support agreement (TS-14-14).

\section{REFERENCES}

[1] F. W. Taylor, "On the art of metal cutting," Trans. ASME, vol. 28, 1907, pp. 31-350. https://doi.org/10.1038/scientificamerican01121907-25942supp

[2] Astakhov, Tribology of Metal Cutting, 2006, Elsevier, San Diego, CA, USA.

[3] V. P. Astakhov, "The assessment of cutting tool wear," International Journal of Machine Tools and Manufacture, vol. 44, no. 6, pp. 637-647, 2004.

https://doi.org/10.1016/j.ijmachtools.2003.11.006

[4] S. E. Oraby and A. M. Alaskari, "On the variability of tool wear and life at disparate operating parameters," Kuw. J. Sci. \& Eng., vol. 35(1B), p. $123,2008$.

[5] Y. Altintas, Manufacturing automation, 2012. Cambridge University Press, Cambridge, UK.

[6] B. S. Prasad et al., "Condition monitoring of CNC machining using adaptive control," International Journal of Automation and Computing, vol. 10, no. 3, pp. 202-209, 2013. https://doi.org/10.1007/s11633-013-0713-1

[7] Y. Huang and Y. Juntang, "High Speed Constant Force Milling Based on Fuzzy Controller and BP Neural Network," International Journal of Control \& Automation, vol. 8, no. 5, p. 143,2014. https://doi.org/10.14257/ijca.2014.7.5.16

[8] S. E. Oraby, E. A. Almeshaiei, and A. Alaskari, "An adaptive control simulation approach based on a mathematical model optimization algorithm for rough turning," Kuwait J. Sci. Eng, vol. 30, no. 2, pp. 213234, 2003.

[9] F. Cus et al., "Adaptive controller design for feedrate maximization of machining process," Journal of Achievements in Materials and Manufacturing Engineering, vol. 17, no.1-2, 2006.

[10] Sun, Yuwen, et al. "A novel adaptive-feedrate interpolation method for NURBS tool path with drive constraints," International Journal of Machine Tools and Manufacture, vol. 77, pp. 74-81, 2014. https://doi.org/10.1016/j.ijmachtools.2013.11.002

[11] http://www.omative.com/173890/ACM

[12] M. S. Alajmi and S. E. Oraby, "On the Influence of the Speed-Feed Interaction on the Wear Rate and Life of Multiple Coated Carbide Inserts Considering Rough Turning Process," Applied Mechanics and Materials, Trans Tech Publications, vol. 575, pp. 431-436, 2014. https://doi.org/10.4028/www.scientific.net/AMM.575.431

[13] S. E. Oraby and D. R. Hayhurst, "Tool life determination based on the measurement of wear and tool force ratio variation," International Journal of Machine Tools and Manufacture, vol. 44, no. 12, 1261-1269, 2004. https://doi.org/10.1016/j.ijmachtools.2004.04.018

[14] S. E. Oraby and D. R. Hayhurst, "High-capacity compact threecomponent cutting force dynamometer," International Journal of Machine Tools and Manufacture, vol. 30, no. 4, pp. 549-559, 1990. https://doi.org/10.1016/0890-6955(90)90006-5 